\title{
From the laboratory to the clinic: How translational studies in animals have lead to clinical advances in boron neutron capture therapy
}

\author{
Rolf F. Barth ${ }^{\mathrm{a}^{*}}$ \\ * Corresponding author: \\ ${ }^{a}$ Rolf F. Barth, M.D. \\ The Ohio State University \\ Department of Pathology \\ 4132 Graves Hall \\ 333 W. 10th Avenue \\ Columbus, OH 43210, U.S.A. \\ Tel: 01-614-292-2177 \\ Email: rolf.barth@osumc.edu
}

\begin{abstract}
In this report five examples have been selected to illustrate how studies in experimental animals have lead directly to clinical implementation. These include (1) the use of BSH as a boron delivery agent for BNCT of patients with brain tumors, and more specifically gliomas; (2) the use of BPA as a delivery agent for BNCT for patients with melanomas and (3) its subsequent use for BNCT of patients with gliomas; (4) optimization of the delivery of BPA in patients with gliomas; and finally (5) the combination of BSH and BPA with BNCT and $\mathrm{X}$-irradiation to treat patients with gliomas.
\end{abstract}

Keywords: sodium borocaptate (BSH), boronophenylalanine (BPA), melanomas and brain tumors, optimization of delivery of BSH and BPA

\section{Introduction of Sodium Borocaptate as a Boron Delivery Agent}

A frequently asked question relating to any therapeutic modality is what lead to its eventual clinical use? The five examples that will be discussed in this short review will demonstrate how studies in experimental animals subsequently resulted in rapid clinical implementation. Those readers without background knowledge of Boron Neutron Capture Therapy are referred to a more comprehensive review on this subject (Barth et al., 2012). The first example relates to the use of sodium borocaptate as a boron delivery agent. The first clinical trials using BNCT to treat patients with high grade gliomas were initiated by Sweet and his co-workers at the Massachusetts General Hospital (MGH) (Asbury et al., 1972), and by Farr and his colleagues at the Brookhaven National Laboratory (BNL) (Farr et al., 1954) using a group of non-tumorselective inorganic boron compounds as delivery agents. The clinical results obtained were disappointing to say the least (Slatkin, 1991). This lead Soloway, Hatanaka and Davis (Soloway et al., 1967) in Sweet's laboratory at the MGH to evaluate a group of tumor-binding sulfhydrylcontaining boron compounds as potential delivery agents. Among these was the sodium salt of the undecahydromercapto-closo-dodecaborate anion $\left(\mathrm{B}_{12} \mathrm{H}_{11} \mathrm{SH}^{2-}\right.$ or $\left.\mathrm{BSH}\right)$. Initially, this compound was evaluated in $\mathrm{C} 3 \mathrm{H}$ mice bearing subcutaneous (s.c.) implants of a murine ependymoblastoma, and the tumor, normal brain and blood values are summarized in Table 1. The enhanced tumor uptake and cell binding properties of BSH lead to a more complete pharmacological and toxicological evaluation in rabbits. It was determined that rapid intravenous 
(i.v.) injection of an isotonic solution of BSH (13.5 $\mathrm{mg}$ boron/ml) was highly toxic, while in contrast, a slow i.v. infusion of a more dilute isotonic solution $(6-7 \mathrm{mg}$ boron/ $\mathrm{ml})$ was well tolerated. Upon his return to Japan, Hatanaka and his co-workers initiated a therapy study in C57BL mice bearing s.c. implants of a transplantable methylcholanthrene-induced sarcoma (Hasegawa et al., 1986). Varying doses of BSH were administered intraperitoneally (i.p.) and the animals were stratified according to the time after administration and the estimated neutron radiation dose. As shown in Fig. 1A, 7 of 13 mice showed no evidence of tumor growth or treatment-related toxicity. Based on these studies, carried out in the late 1960s, Hatanaka initiated a clinical trial in patients with high grade gliomas who had undergone surgical resection of their tumors, which then was followed by BNCT using BSH as the delivery agent. The results of this early study in patients with brain tumors are summarized in Fig. 1B (Hatanaka, 1991). Although there were a number of challenges relating to the clinical implementation of BNCT, the treatment was well tolerated and the patients appeared to have prolonged survival times compared to historical controls. These early pioneering studies lead to more extensive clinical studies by Hatanaka and Nakagawa (1994) to further evaluate the efficacy of BNCT to treat patients with a variety of brain tumors, the majority of which had high grade gliomas. However, due to the fact that these were non-randomized studies using historical controls, the results were viewed with some skepticism. Nevertheless, they established the use of BSH as a clinical boron delivery agent.

\section{Development of Boronophenylalanine as a Boron Delivery Agent for BNCT of Melanomas}

Mishima, a Japanese dermatologist, and his colleagues (Mishima et al., 1989a, b) at Kobe University had a special interest in the possibility of using BNCT to treat patients with cutaneous melanomas who, for one or another reason, were not considered to be candidates for surgery. Initial in vitro studies were carried out with boron-10 containing analogues of chlorpromazine, which had been shown to selectively bind to melanin. Mishima et al. (1989b) then focused their attention on a boron-containing precursor of melanin, a tyrosine substrate, ${ }^{10} \mathrm{~B}$ paraboronophenylalanine (designated boronophenylalanine or "BPA"), which they hypothesized would be taken up by melanin-producing tumor cells. In vitro radiobiologic studies with BPA yielded promising data and based on these results, in vivo studies were carried out using animal models for spontaneously occurring melanomas in Syrian hamsters and then in Duroc pigs (Mishima et al., 1989b). BPA was administered perilesionally by two s.c. injections, each of $5 \mathrm{~g}$ of the compound, the first dose 19 hours before irradiation of the pig and the second 20 minutes prior to irradiation with a collimated beam of thermal neutrons at the Kyoto University Research Reactor Institute (KURRI). As shown in Fig. 2A, prior to irradiation, and in Fig. 2B, after BNCT, there was complete regression of the tumor at 115 days following treatment. Based on this unequivocal demonstration of therapeutic efficacy, a clinical trial was initiated. The first patient treated was a 60 -year-old male who had a primary acral lentiginous melanoma on the sole of his right foot. Five injections of BPA, as a fructose complex to increase its water solubility (Yoshino et al., 1989), were administered perilesionally into his right sole over 19 hours, and 2 hours after the first injection the patient was irradiated at KURRI (Mishima et al., 1989a). Complete regression was observed at 9 months, and this success was followed by other patients with a variety of cutaneous melanomas, one of whom was an 85-year-old woman with a nodular melanoma of the left foot (Fig. 3A) (Mishima, 1996). She received a total of $170 \mathrm{mg}$ of BPAfructose complex per $\mathrm{kg}$ body weight injected perilesionally at a distance of $4 \mathrm{~cm}$ from the 

disappearance at 6 months (Fig. 3C). These results unequivocally established the clinical efficacy of BNCT and lead the way to the next advance.

\section{Demonstration that BPA Could Be Used to Treat Brain Tumors}

Shortly after Mishima's reports that BPA could be used as a boron delivery agent for BNCT of melanomas, Coderre and his co-workers at BNL initiated studies to further evaluate BPA, which was administered intragastrically by gavage. Initially, biodistribution studies were carried out and these demonstrated that BPA could deliver potentially therapeutic amounts of boron to Harding-Passey melanoma bearing mice (Coderre et al,. 1988) and subsequently to mice bearing a variety of other tumor types (Coderre et al., 1990). These included mice bearing s.c. implants of the KHJJ murine mammary carcinoma and rats bearing intracerebral implants of the 9L gliosarcoma (Coderre et al., 1990). These biodistribution studies were followed by a therapy study (Coderre et al., 1992) demonstrating that BPA, administered to Fischer rats bearing intracerebral implants of the 9L gliosarcoma, had significantly longer median survival times following BNCT at the Brookhaven Medical Research Reactor (BMRR). Kaplan-Meier survival plots are shown in Fig. 4A, with $50 \%$ of the animals alive at one year compared to those treated with $250 \mathrm{kVp}$ X-rays. These studies firmly laid the groundwork for a Phase I clinical biodistribution study by Elowitz et al. (1998) using a 2-hour i.v. infusion of BPA-fructose. A group of 17 patients were studied and it was noted that the blood boron concentrations were proportional to the dose of BPA administered. The normal brain concentrations generally were equal to or less than that in the blood. Tumor boron concentrations on the other hand were highly variable but were always greater than those of the blood and normal brain. This study provided the basis for a Phase I/II clinical trial by the Brookhaven group (Chanana et al., 1999) involving 38 patients with glioblastomas. BNCT was shown to be safe and, as illustrated in Fig. 4B, the overall survival of patients following a single application of BNCT was comparable to that obtained with a standard 7-8 week course of fractionated photon therapy.

\section{Optimization of the Delivery of BPA}

In order to improve the tumor uptake of BPA, a collaborative study was initiated by Smith (Smith et al., 2001) et al. at Cornell University, Barth and Yang at The Ohio State University (OSU), and Joel, Coderre and Morris et al. (2002) at BNL using the F98 and 9L glioma models, respectively. Rats were stratified into groups that received BPA by i.v. injection, intracarotid (i.c.) injection either alone or in combination with blood-barrier disruption (BBB-D) by means of an i.c. infusion of a hyperosmotic solution of mannitol (Barth et al., 1997). Intravenous infusion times of BPA ranged from 2 hours to 6 hours. It was shown by secondary ion mass spectrometry (SIMS) that rats which had received a 6 hour i.v. infusion of BPA, the boron-10 concentration in infiltrating tumor cells had increased by nearly $90 \%$ relative to that observed following 2 and 3 hour infusions (Smith et al., 2001) (Fig. 5A). BBB-D in F98 glioma bearing rats resulted in a 2.45 -fold increase in the tumor to normal brain boron concentration ratios, with even higher concentrations in infiltrating tumor cells. Based on the experimental data demonstrating a significant increase $(\mathrm{P}<0.05)$ in boron uptake in tumor cell clusters following a 6-hour vs a 2-hour i.v. infusion of BPA with a $90 \%$ increase in boron concentrations $(65 \pm 11$ vs $31 \pm 12 \mu \mathrm{g}$ B/g tumor) (Smith et al., 2001), a Phase II clinical trial was initiated by a group in Studsvik, Sweden to evaluate a 6 hour infusion of BPA in patients with glioblastomas (Capala et al., 2003; Sköld et al., 2010). The Kaplan-Meier survival plots for these two groups are shown in Fig. 5B. 
Although some of the patients in both arms of the study had received temozolomide, nevertheless the overall median survival time for those patients who had received BNCT was 17.6 months versus 12.9 months for radiation therapy alone. Local control of GBM was achieved in the Studsvik study but not the BNL, as shown by neuropathlogical examination following death of the patients. Based on these results it was suggested that a 6 hour i.v. infusion of BPAfructose followed by BNCT should be further evaluated (Hopewell et al., 2011).

\section{Combination of BSH and BPA with BNCT Alone or in Combination with X-irradiation}

The last example that has been selected relates to studies carried out by Barth and his coworkers at OSU and Ono and his research team in Japan at KURRI to evaluate the therapeutic efficacy of BSH and BPA with BNCT either alone or in combination with X-irradiation. Using the F98 rat glioma model, Barth et al. evaluated BSH and BPA, administered i.v., i.c., or i.c. together with BBB-D (Barth et al., 2000). As shown in the Kaplan-Meier survival plots (Fig. 6A), the combination of BSH and BPA administered i.v. was superior to BPA alone (Barth et al., 1997 and Barth et al., 2000). Enhanced survival was seen if both agents were administered i.c. and this was further improved with BBB-D disruption. The corresponding mean survival times (MSTs) were 41, 73, and 140 days, respectively, with 25\% long-term survivors (>180 d). Using the SCCCVIII murine tumor model, Ono and his research team (Ono et al., 1999) at KURRI showed that, following BNCT, higher tumor control was achieved using the combination of i.v. BSH and BPA. As illustrated in Fig. 6B, when tumor control probability was plotted as a function of the estimated radiation dose $(\mathrm{Gy}), 50 \%$ of the tumors were controlled $\left(\mathrm{TCD}_{50}\right)$ at 13.4 Gy with BPA and 15.9 Gy with BSH. When BSH and BPA were combined the $\mathrm{TCD}_{50}$ was 8.4 Gy. Based on these studies, Miyatake and Kawabata at the Osaka Medical College and their co-workers initiated a clinical trial involving 22 patients to evaluate the efficacy of BSH and BPA in combination with one another for the treatment of patients with recurrent malignant gliomas (Miyatake et al., 2009). The MST for all patients was 10.8 months, and for those patients with glioblastomas (GBM) it was 9.6 months after recurrence and 19.1 months after initial diagnosis (Fig. 7A). Although local control of recurrent malignant gliomas was fairly good, it is noteworthy that a subset of patients had spinal cord dissemination of their tumors at the time of death. The cause of this has yet to be determined but it provides a cautionary note. Based in part on Barth et al.'s (2004) and Ono et al.'s (1999) data, which demonstrated that BSH was distributed homogeneously and BPA heterogeneously within tumors, Suzuki and Kato and their clinical team (Suzuki et al., 2014) initiated a clinical trial using a combination of BSH and BPA to treat patients with recurrent tumors of the head and neck region. A total of 72 patients were treated, 15 of whom received $\mathrm{BSH}(5 \mathrm{~g})$ and BPA-fructose $(250 \mathrm{mg} / \mathrm{kg})$. No comparison could be made in the effectiveness of using this combination due to heterogeneity of the tumor types that were treated, but there were some striking clinical responses (Barth et al., 2012; Suzuki et al., 2014).

A second component of the studies of Barth et al. (2004) related to combining BNCT with an external beam photon boost. F98 glioma bearing rats that had received $\mathrm{BSH}$ and BPA were divided into two groups, one of which received BNCT alone, and the other BNCT in combination with 15 Gy of $6 \mathrm{MV}$ photons, produced by a linear accelerator and delivered in three consecutive daily fractions of $5 \mathrm{~Gy}$ each. These groups of animals all had significant increases in their MSTs, including those that received i.v. BSH and BPA (Fig. 6A). Based on these studies Kawabata and Miyatake and his clinical team (Kawabata et al., 2009) initiated a 

followed by BNCT and two weeks later an X-ray boost of 20 to $30 \mathrm{~Gy}$ administered daily in 2 Gy fractions. As shown in the Kaplan-Meier survival plot (Fig. 7B), a MST of 23.5 months was observed in the group that received the photon boost, with 11 patients still alive at the time of publication, which are the best survival data yet reported in patients with high grade gliomas who have been treated with BNCT.

\section{Conclusions}

The five examples presented in this report demonstrate how experimental animal data resulted in significant clinical advances in the application of BNCT. However, a cautionary note is that what might be feasible in experimental animals could be very difficult if not impossible to carry out in a clinical setting (Barth and Coderre, 2003). The first example relates to studies using the F98 rat glioma model to optimize the delivery of BSH and BPA by administering these drugs by i.c. injection with or without BBB-D (Barth et al., 2000). Although i.c. administration of cytoreductive chemotherapeutic agents has been used by Neuwelt and his clinical team (Angelov et al., 2009), this is a challenging procedure that probably could not be carried out in a clinical BNCT facility. The second is direct intracerebral administration by convection enhanced delivery (CED) of boron-containing, tumor-targeting therapeutic agents such as boronated porphyrins (Kawabata et al., 2011), anti-EGFR monoclonal antibodies (Yang et al., 2008), or thymidine kinase (TK) 1 targeting carboranyl thymidine analogues (Barth et al., 2008).

Where do we go from here? First, and perhaps foremost, there is a need to develop new boron delivery agents. This has been an oft-stated need for more than 25 years, and yet there still are no new agents that have progressed from evaluation in experimental animals to clinical biodistribution studies. There are a number of reasons for this, but one of the most important has been the lack of convincing experimental animal data that would warrant the initiation of expensive clinical biodistribution studies that would not directly benefit the patients participating in these studies. Second, further studies to optimize the dosing paradigm of BSH and BPA could be carried out without the need of obtaining permission of regulatory agencies, which would be necessary for new boron delivery agents. Although some of the clinical results obtained with BNCT have been impressive, it remains to be determined if it will gain acceptance among a broader group of physicians who are taking care of cancer patients on a day-to-day basis.

\section{Acknowledgments}

This paper is dedicated to Professor Emeritus Albert Soloway in celebration of his 90th birthday. The studies carried out by Dr. Barth and his co-workers were supported by grants from the National Institutes of Health and the United States Department of Energy. The secretarial assistance of Loretta Bahn in the preparation of this report is gratefully acknowledged.

\section{References}

Angelov, L., Doolittle, N.D., Kraemer, D.F., Siegal, T., Barnett, G.H., Peereboom, D.M., Stevens, G., McGregor, J., Jahnke, K., Lacy, C.A., Hedrick, N.A., Shalom, E., Ference, S., Bell, S., Sorenson, L., Tyson, R.M., Haluska, M., Neuwelt, E.A. 2009. Blood-brain barrier disruption and intra-arterial methotrexate-based therapy for newly diagnosed primary CNS lymphoma: A multiinstitutional experience. J Clin. Oncol. 27, 3503-3509. DOI 10.1200/JCO.2008.19.3789. 
Asbury, A.K., Ojemann, R.G., Nielsen, S.L., Sweet, W.H. 1972. Neuropathologic study of fourteen cases of malignant brain tumor treated by boron-10 slow neutron capture radiation. $\mathrm{J}$. Neuropath. Exp. Neurol. 31, 278-303.

Barth, R.F., Grecula, J.C., Yang, W., Rotaru, J.H., Nawrocky, M., Gupta, N., Albertson, B.J., Ferketich, A.K., Moeschberger, M.L., Coderre, J., Rofstad, E.K. 2004. Combination of boron neutron capture therapy and external beam radiotherapy for brain tumors. Int'l J. Radiat. Oncol. Biol. \& Physics 58, 267-277.

Barth, R.F., Vicente, M.G., Harling, O.K., Riley, K.J., Binns, P.J., Kiger III, W.S., Wagner, F.M., Suzuki, M., Aihara, T., Kato, T., Kawabata, S.: Current status of boron neutron capture therapy of high grade gliomas and recurrent head and neck cancer. Radiation Oncology 7:146$166,2012$.

Barth, R.F., Yang, W., Coderre, J.A. 2003. Rat brain tumor models to assess the efficacy of boron neutron capture therapy: A critical evaluation. J. Neuro-Oncology 62: 61-74.

Barth, R.F., Yang, W., Rotaru, J.H., Moeschberger, M.L., Boesel, C.P., Soloway, A.H., Joel, D.D., Nawrocky, M.M., Ono, K., Goodman, J.H. 2000. Boron neutron capture therapy of brain tumors: Enhanced survival and cure following blood-brain barrier disruption and intracarotid injection of sodium borocaptate and boronophenylalanine. Intl. J. Radiat. Oncol. Biol. \& Physics 47, 209218.

Barth, R.F., Yang, W., Rotaru, J.H., Moeschberger, M.L., Joel, D.D., Nawrocky, M.M., Goodman, J.H., Soloway, A.H. 1997. Boron neutron capture therapy of brain tumors: enhanced survival following intracarotid injection of either sodium borocaptate or boronophenylalanine with or without blood-brain barrier disruption. Cancer Res 57, 1129-1136.

Barth, R.F., Yang, W., Wu, G., Swindall, M.R., Byun, Y., Narayanasamy, S., Tjarks, W., Tordoff, K., Moeschberger, M., Eriksson, S., Binns, P.J., Riley, K.J. 2008. Thymidine kinase 1 as a molecular target for boron neutron capture therapy of brain tumors. Proc. Nat'l. Acad. Sciences 105, 17493-17497.

Capala, J., H.-Stenstam, B., Sköld, K., Munck af Rosenschöld, P., Giusti, V., Persson, C., Wallin, E., Brun, A., Franzen, L., Carlsson, J., Salford, L., Ceberg, C., Persson, B., Pellettiere, L., Henriksson, R. 2003. Boron neutron capture therapy for glioblastoma multiforme: Clinical studies in Sweden. J. Neurooncol. 62, 135-144.

Chanana, A.D., Capala, J., Chadha, M., Coderre, J.A., Diaz, A.Z., Elowitz, E.H., Iwai, J., Joel, D.D., Liu, H.B., Ma, R., Pendzick, N., Perress, N.S., Shady, M.S., Slatkin, D.N., Tyson, G.W., Wielopolski, L. 1999. Boron neutron capture therapy for glioblastoma multiforme: Interim results from the Phase I/II dose-escalation studies. Neurosurgery 44, 1182-1193.

Coderre, J.A., Glass, J.D., Fairchild, R.G., Micca, P.L., Fand, I., Joel, D.D. 1990. Selective delivery of boron by the melanin precursor analogue $p$-boronophenylalanine to tumors other than melanoma. Cancer Res. 50, 138-141. 

intracerebral gliosarcomas in rats by boron neutron capture therapy with $p$-boronophenylalanine. Radiat. Res. 129, 290-296.

Coderre, J.A., Kalef-Ezra, J.A., Fairchild, R.G., Micca, P.L., Reinstein, L.E., Glass, J.D. 1988. Boron neutron capture therapy of a murine melanoma. Cancer Res. 48, 6313-6316.

Elowitz, E.H., Bergland, R.M., Coderre, J.A., Joel, D.D., Chadha, M., Chanana, A.D. 1998. Biodistribution of $p$-boronophenylalanine in patients with glioblastoma multiforme for use in boron neutron capture therapy. Neurosurgery 42, 463-469.

Farr, L.E., Sweet, W.H., Robertson, J.S., Foster, C.G., Locksley, H.B., Sutherland, D.L., Mendelsohn, M.L., Stickley, E.E. 1954. Neutron capture therapy with boron in the treatment of glioblastoma multiforme. Amer. J. Roentgenol., Radium Ther. Nuclear Med. 71, 279-291.

Hasegawa, H., Mogami, H., Amano, K., Kamano, S., Hatanaka, H. 1986. Boron neutron therapy for transplantable mouse gliomas in 1974. In H. Hatanaka, Ed., Neutron Capture Therapy, Nishimuro Co. Ltd., pp. 419-430.

Hatanaka, H. 1991. Boron-neutron capture therapy for tumors in glioma. In A.B.M.F. Karim and E.R. Laws, Jr., Eds., Glioma. Springer-Verlag, Berlin. pp. 233-249.

Hatanaka, H., Nakagawa, Y. 1994. Clinical results of long-surviving brain tumor patients who underwent boron neutron capture therapy. Int. J. Radiat. Oncol. Biol. Phys. 28, 1061-1066.

Hopewell, J.W., Gorlia, T., Pellettieri, L., Giusti, V., H.-Stenstam, B., Sköld, K. 2011. Boron neutron capture therapy for newly diagnosed glioblastoma multiforme: An assessment of clinical potential. Appl. Radiat. Isot. 69, 1737-1740.

Kawabata, S., Miyatake, S.-I., Hiramatsu, R., Hirota, Y., Miyata, S., Takekita, Y., Kuroiwa, T., Kirihata, M., Kakurai, Y., Maruhashi, A., Ono, K. 2011. Phase II clinical study of boron neutron capture therapy combined with X-ray radiotherapy/temozolomide in patients with newly diagnosed glioblastoma multiforme - Study design and current status report. Appl. Radiat. Isot. 69, 1796-1799. DOI 10.1016/j.apradiso.2011.03.014.

Kawabata, S., Miyatake, S.-I., Kuroiwa, T., Yokoyama, K., Doi, A., Iida, K., Miyata, S., Nonoguchi, N., Michiue, H., Takahashi, M., Inomata, T., Imahori, Y., Kirihata, M., Sakurai, Y., Maruhashi, A., Kumada, H., Ono, K. 2009. Boron neutron capture therapy for newly diagnosed glioblastoma. J. Radiat. Res. 50, 51-60.

Kawabata S., Yang, W., Barth, R.F., Wu, G., Huo, T., Binns, P.J., Riley, K.J., Ongayi, O., Gottumukkala, V., Vicente, M.G.H. 2011. Convection enhanced delivery of carboranylporphyrins for neutron capture therapy of brain tumors. J. Neuro-Oncology 103:175-185.

Mishima, Y. 1996. Selective thermal neutron capture therapy of cancer cells using their specific metabolic activities - melanoma as a prototype. In Cancer Neutron Capture Therapy, Mishima, Y., Ed. Plenum Press, New York, NY. pp. 1-26. 

Kobayashi, T., Kanda, K., Yoshino, K. 1989a. Treatment of malignant melanoma by single neutron capture therapy with melanoma-seeking ${ }^{10} \mathrm{~B}$-compound. Lancet 1, 388-389.

Mishima, Y., Ichihashi, M., Tsuji, M., Hatta, S., Ueda, M., Honda, C., Suzuki, T. Treatment of malignant melanoma by selective thermal neutron capture therapy using melanoma-seeking compound. 1989b. J. Invest. Dermatol. 92, 321S-325S.

Miyatake, S.-I., Kawabata, S., Yokoyama, K., Kuroiwa, T., Michiue, H., Sakurai, Y., Kumada, H., Suzuki, M., Maruhashi, A., Kirihata, M., Ono, K. 2009. Survival benefit of boron neutron capture therapy for recurrent malignant gliomas. J. Neuro-oncol. 91, 199-206. DOI $10.1007 / \mathrm{s} 11060-008-9699-\mathrm{x}$.

Morris, G.M., Micca, P.L., Nawrocky, M.M., Weissfloch, L.E., Coderre, J.A. 2002. Long-term infusions of $p$-boronophenylalanine for boron neutron capture therapy: Evaluation using rat brain tumor and spinal cord models. Radiat. Res. 158, 743-752.

Ono, K., Masunaga, S.-I., Suzuki, M., Kinashi, Y., Takagaki, M., Akaboshi, M. 1999. The combined effect of boronophenylalanine and borocaptate in boron neutron capture therapy for SCCVII tumors in mice. Int. J. Radiat. Oncology Biol. Phys. 43, 431-436.

Sköld, K., Gorlia, T., Pellettieri, L., Giusti, V., H-Stenstam, B., Hopewell, J.W. 2010. Boron neutron capture therapy for newly diagnosed glioblastoma multiforme: An assessment of clinical potential. The Brit. J. Radiol. 83, 596-603.

Slatkin, D.N. 1991. A history of boron neutron capture therapy of brain tumours: Postulation of a brain radiation dose tolerance limit. Brain 114, 1609-1629.

Smith, D.R., Chandra, S., Barth, R.F., Yang, W., Joel, D.D., Coderre, J.A. 2001 . Quantitative imaging and microlocalization of boron-10 in brain tumors and infiltrating tumor cells by SIMS ion microscopy: Relevance to neutron capture therapy. Cancer Res. 61, 8179-8187.

Soloway, A.H., Hatanaka, H., Davis, M.A. 1967. Penetration of brain and brain tumor. VII. Tumor-binding sulfhydryl boron compounds. J. Med. Chem. 10, 714-717.

Suzuki, M., Kato, I., Aihara, T., Hiratsuka, J., Yoshimura, K., Niimi, M., Kimura, Y., Ariyoshi, Y., Haginomori, S.-I., Kakurai, Y., Kinashi, Y., Masunaga, S.-I., Fukushima, M., Ono, K., Maruhashi, A. 2014. Boron neutron capture therapy outcomes for advanced or recurrent head and neck cancer. J. Radiat. Res. 54, 146-153. DOI 10.1093/jrr/rrt098.

Yang, W., Wu, G., Barth, R.F., Swindall, M.R., Bandyopadhyaya, A.K., Tjarks, W., Tordoff, K., Moeschberger, M., Sferra, T.J., Binns, P.J., Riley, K.J., Ciesielski, M.J., Fenstermaker, R.A., Wikstrand, C. 2008. Molecular targeting and treatment of composite EGFR and EGFRvIIIpositive gliomas using boronated monoclonal antibodies. Clin. Cancer Res. 14, 883-891.

Yoshino, K., Suzuki, A., Mori, Y., Kakihana, H., Honda, C., Mishima, Y., Kobayashi, T., Kanda, K. 1989. Improvement of solubility of $p$-boronophenylalanine by complex formation with monosaccharides. Strahlenther Onkol. 165, 127-129. 
Table 1. Biodistribution of Sodium Borocaptate $\left(\mathrm{Na}_{2} \mathrm{~B}_{12} \mathrm{H}_{11} \mathrm{SH}\right.$ or "BSH") in a Murine Ependymoblastoma

\begin{tabular}{|c|c|c|c|c|c|}
\hline \multicolumn{2}{|c|}{ Tumor } & \multicolumn{2}{|c|}{ Blood } & \multicolumn{2}{|c|}{ Brain } \\
\hline Mean & Range & Mean & Range & Mean & Range \\
\hline $21 \pm 17.8$ & $4.8-61.5$ & $2.9 \pm 1.8$ & $1.0-7.2$ & $0.9 \pm 0.5$ & $<0.5-2.0$ \\
\hline
\end{tabular}

*Total dose ranged from $140-175 \mu \mathrm{g}$ boron/g.

Data taken from Penetration of Brain and Brain Tumor. VII. Tumor-Binding Sulfhydryl Boron Compounds. Soloway, A.H., Hatanaka, H., and Davis, M.A., Journal of Medicinal Chemistry 10:714, 1967.

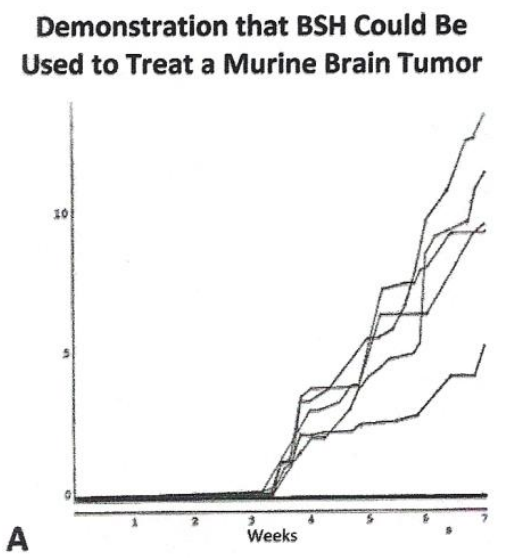

Demonstration that BSH Could be Used Clinically to Treat Patients with Gliomas

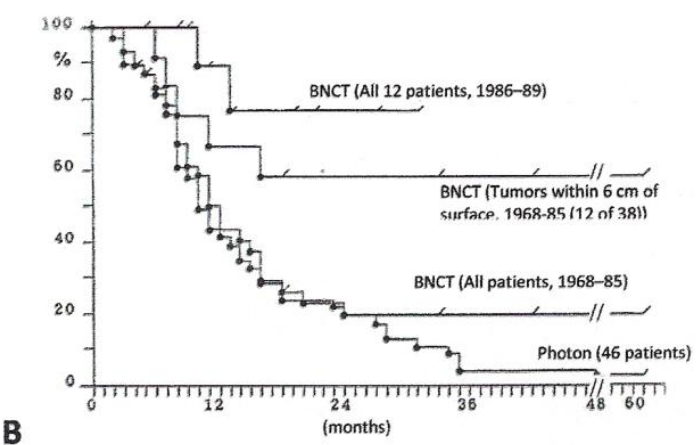

Fig. 1. A. Dose of BSH $100 \mathrm{mg}{ }^{10} \mathrm{~B} / \mathrm{kg}$ i.p. BNCT was initiated $14.5 \mathrm{hr}$ later with an estimated radiation dose of 1,200 rad (cGy). From Hasegawa et al., Boron neutron therapy for transplantable mouse gliomas in 1974. Neutron Capture Therapy. B. Survival of patients with grade III or IV supratentorial glioma (excluding those who had been previously treated; as of 30 Sept 1989). The MST was approximately 44 months and MeST survival was 25.6 months and the 5 year survival was 58.3\%. From Hatanaka, Boron-Neutron Capture Therapy for Tumors, in Glioma.

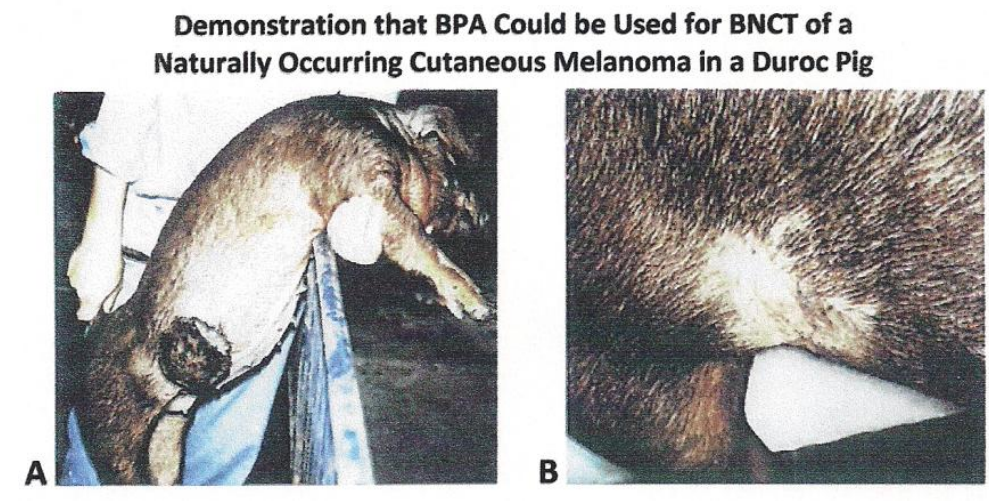

Fig. 2. A. Clinical appearance before BNCT. B. $115 \mathrm{~d}$ after BNCT there was complete disappearance of the melanoma. From Mishima et al., J. Investigative Dermatology, 92(5), 321S-325S. 
Demonstration that BNCT Could Be Used to Treat an Invasive Melanoma of the Foot

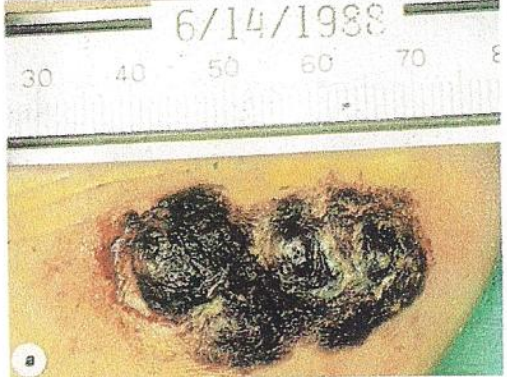

A. Before BNCT

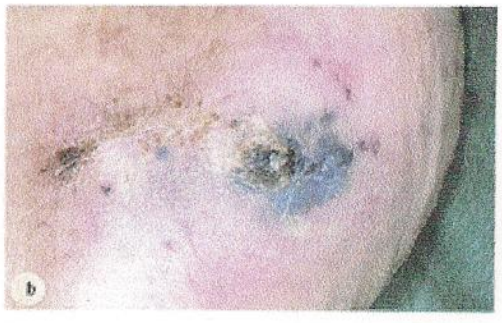

B. 4.5 months after BNCT

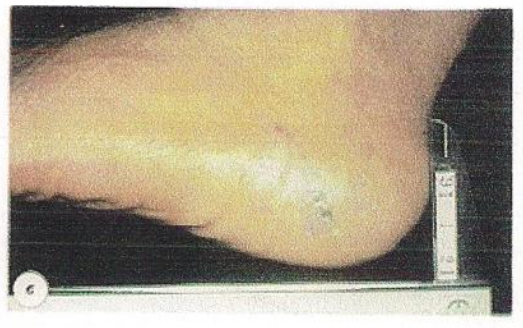

C. 2.5 years after BNCT

Fig. 3. The patient, an 85-year-old woman with a plantar melanoma, was not a candidate for surgery due to a cardiac problem. She received BNCT using BPA as a fructose complex $(170 \mathrm{mg} / \mathrm{kg})$, injected perilesionally. As shown in the photos, there was complete regression without recurrence. From Mishima in Cancer Neutron Capture Therapy, Y. Mishima, Ed. Plenum Press, 1996.

\section{Demonstration that BPA Could be Used as a Delivery Agent to Treat the Rat $9 \mathrm{~L}$ Gliosarcoma}

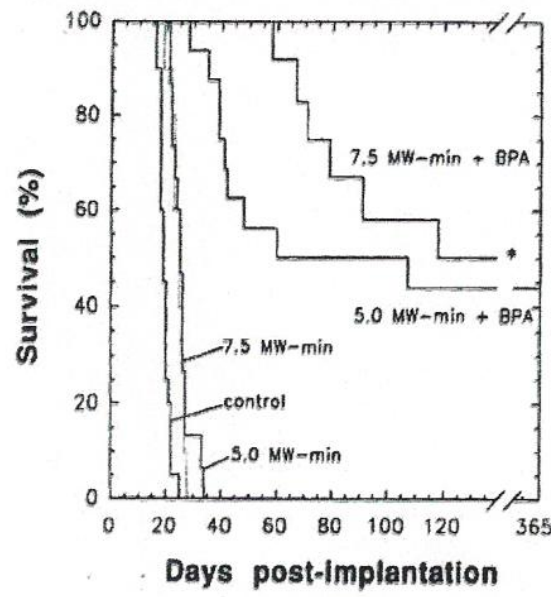

\section{Demonstration that BPA Could be Used Clinically to Treat Patients with GBMs}

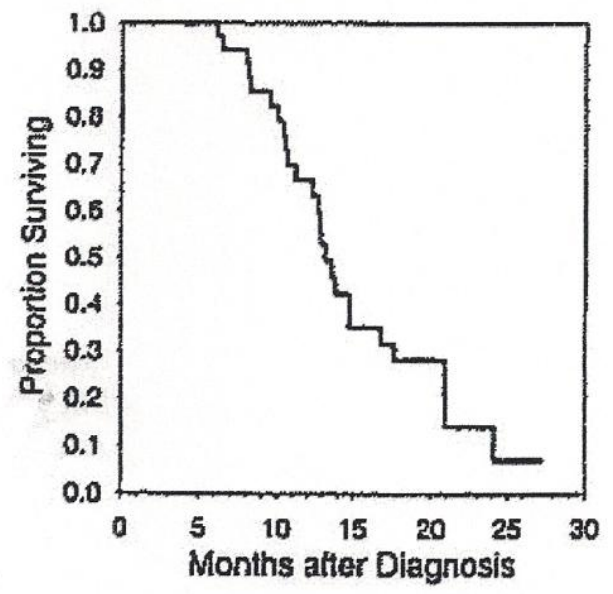

Fig. 4. A. Survival of control and BNCT-treated rats. From Coderre, J.A., Joel, D.D., Micca, P.L., Radiation Research 129:290-296, 1992. B. Overall survival times for 37 patients from time of tissue diagnosis. From Chanana et al., Neurosurgery, 44:1182-1192, 1999. 


\section{Demonstration that Prolonged Infusion} of BPA was Superior to a Short Infusion

A

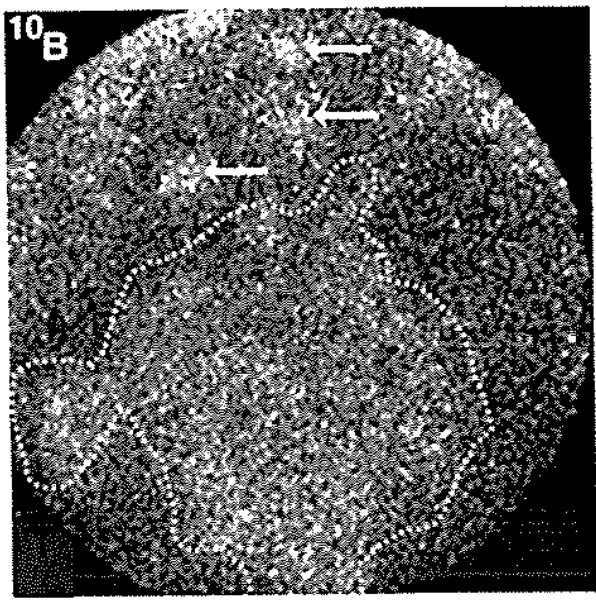

Demonstration that Prolonged Infusion of BPA Resulted in Improved Patient Survival

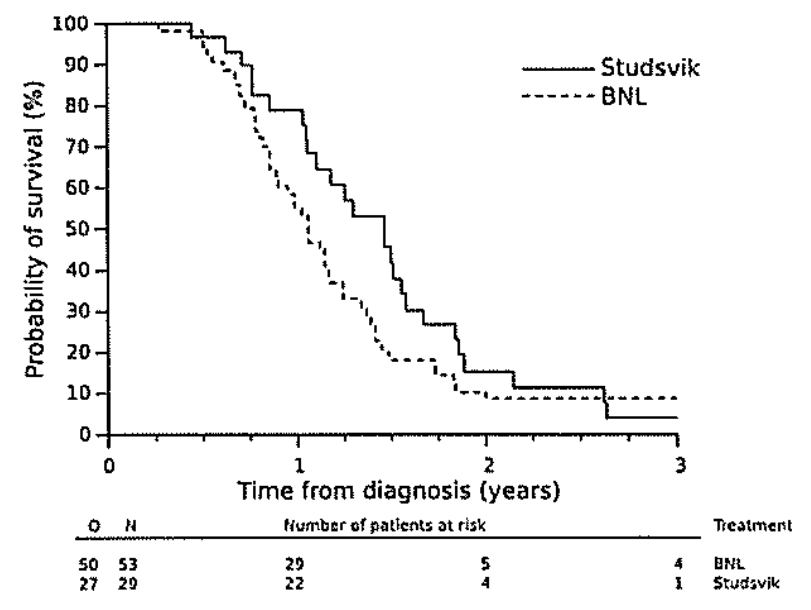

Fig. 5. A. ${ }^{10} \mathrm{~B}$ distribution in the main tumor mass (MTM) and clusters of tumor cells infiltrating normal brain of $\mathrm{F} 98$ glioma bearing rats. After a $6 \mathrm{hr}$ infusion the ${ }^{10} \mathrm{~B}$ concentration in infiltrating tumor cells had increased by almost 90\% relative to 2 or $3 \mathrm{hr}$ infusion. From Smith, D.R., Chandra, S., Barth, R.F. et al., Cancer Res. 61:8179-8187, 2001. B. Kaplan-Meier plots of overall survival for the two BNCT studies. Improved clinical efficacy with prolonged infusion was indicated by a longer MST (17.7 mos) compared to the Brookhaven study (12.6 mos). From Sköld, K., H-Stenstam, B., Diaz, A.Z. et al., Acta Neurol. Scand. 122:58-62, 2010.

Combined Effect of BSH and BPA for BNCT of F98 Glioma Bearing Rats

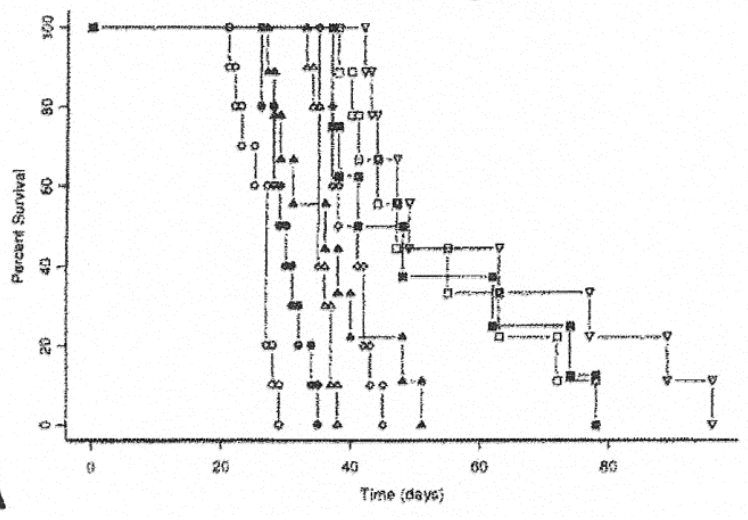

\section{Combined Effect of BSH and BPA for BNCT of SCCVII Murine Tumors}

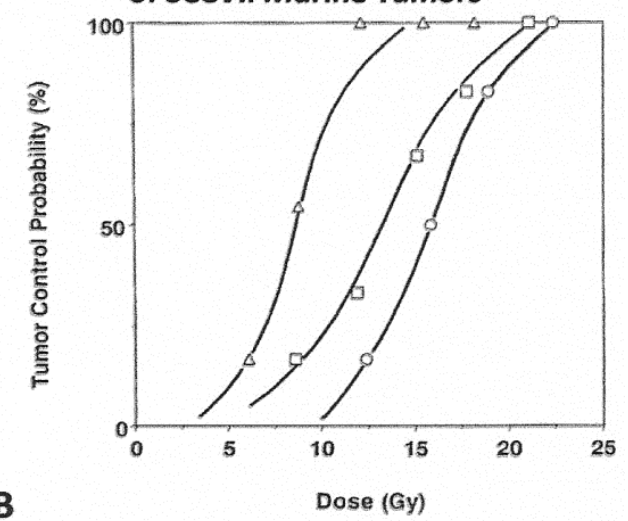

Fig. 6. A. Survival time in days after implantation given for untreated (o); neutrons alone $(\bullet)$; $X$-rays alone $(\triangle)$; neutrons + X-rays $(\boldsymbol{\Delta})$; i.v. BPA + BSH + BNCT $(\diamond)$; intracarotid BPA + BSH + BNCT $(\square)$; i.v. BPA + BSH + BNCT + Xrays ( $)$; and intracarotid $B P A+B S H+B N C T+X$-rays $(\nabla)$. BPA = boron phenylalanine; $B S H=$ sodium borocaptate; BNCT = boron neutron capture therapy. From Barth et al., Int. J. Radiation Oncology Biol. Phys. 58:267-277, 2004. B. Tumor control rates as a function of radiation dose in Gy. (): BPA $1500 \mathrm{mg} / \mathrm{kg}\left(58.6 \mathrm{mg}{ }^{10} \mathrm{~B} / \mathrm{kg}\right.$ ), (O): BSH $75 \mathrm{mg} / \mathrm{kg}\left(41.0 \mathrm{mg}{ }^{10} \mathrm{~B} / \mathrm{kg}\right),(\triangle)$ : BPA $\left(750 \mathrm{mg} / \mathrm{kg}: 29.3 \mathrm{mg}{ }^{10} \mathrm{~B} / \mathrm{kg}\right)+\mathrm{BSH}(75 \mathrm{mg} / \mathrm{kg})$. From Ono et al., Int. J. Radiat. Oncol. Biol. Phys. 43:431-436, 1999. 
Kaplan-Meier Survival Plots of Glioma Patients Treated with BNCT Alone or in Combination with X-Irradiation
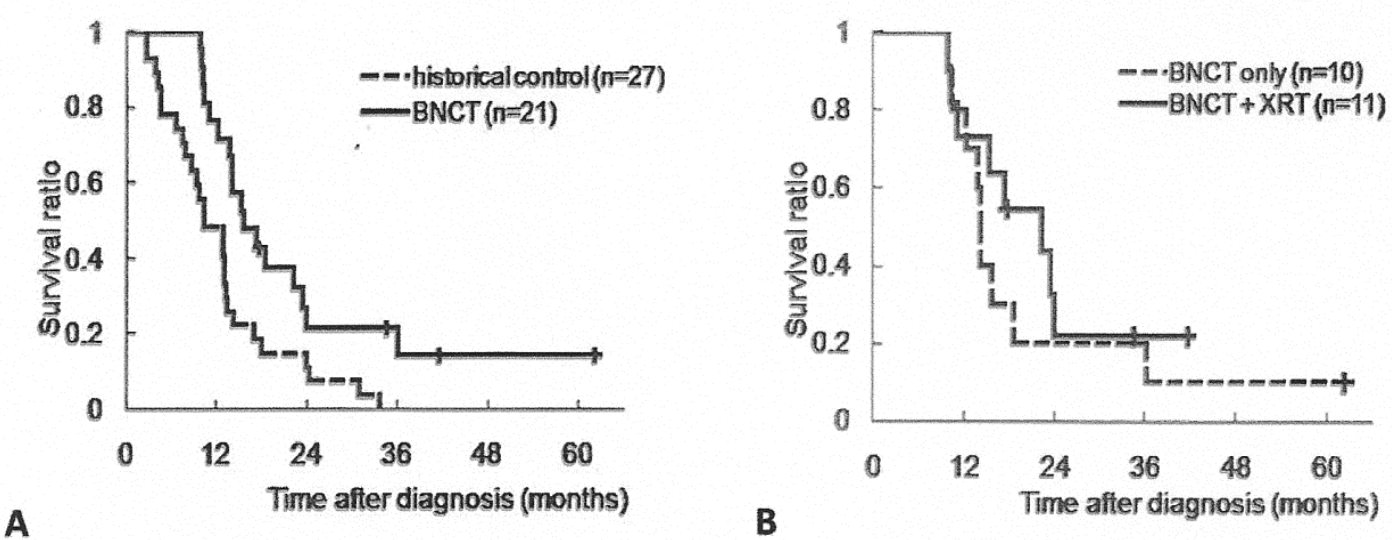

Fig. 7. A. Survival times of the patients treated with BNCT (protocols 1 plus 2, $n=21$ ), MST 15.6 months (-). Survival times of our institutional historical controls (- -) (surgical removal, XRT, and chemotherapy) ( $n=27)$, MST 10.3 months (log-rank test, $p=0.0035$ ). B: Survival times of the patients treated with BNCT followed by XRT boost (protocol 2, n=11), MST 23.5 months (-). Survival times of the patients treated by protocol $1(n=10)$, MST $14.1(--)$. However, there was no statistical significance in the difference in MSTs between these groups. From Kawabata, S., Miyatake, S-I., et al. J. Radiat. Res. 50:51-60, 2009. 